\title{
Low-threshold photonic crystal laser
}

\author{
Marko Lončar, a) Tomoyuki Yoshie, and Axel Scherer \\ Department of Electrical Engineering, California Institute of Technology, MS 136-93, Pasadena, \\ California 91125 \\ Pawan Gogna and Yueming Qiu \\ In Situ Technology and Experiments Systems Section, Jet Propulsion Laboratory, California Institute of \\ Technology, MS 302-306, 4800 Oak Grove Drive, Pasadena, California 91109
}

(Received 19 June 2002; accepted 9 August 2002)

\begin{abstract}
We have fabricated photonic crystal nanocavity lasers, based on a high-quality factor design that incorporates fractional edge dislocations. Lasers with InGaAsP quantum well active material emitting at $1550 \mathrm{~nm}$ were optically pumped with $10 \mathrm{~ns}$ pulses, and lased at threshold pumping powers below $220 \mu \mathrm{W}$, the lowest reported for quantum-well based photonic crystal lasers, to our knowledge. Polarization characteristics and lithographic tuning properties were found to be in excellent agreement with theoretical predictions. (C) 2002 American Institute of Physics.
\end{abstract}

[DOI: $10.1063 / 1.1511538]$

The quest for a compact nanocavity laser, with highquality factor $(Q)$ and small mode volume $\left(V_{\text {mode }}\right)$, has been a central part of research in the field of integrated optics. Photonic crystals, ${ }^{1}$ and planar photonic crystals in particular $(\mathrm{PPC}),{ }^{2}$ are promising manufacturable geometries for the realization of compact optical nanocavities and their integration with waveguides, modulators, and detectors. So far, there have been several reports on room-temperature lasing in PPC nanocavities, ${ }^{3-7}$ and more recently, new high- $Q$ cavity designs based on modification of two-dimensional (2D) photonic crystals have been proposed. ${ }^{4,8}$ In this letter, we report the experimental application of one of these designs. The cavities are based on fractional edge dislocations, ${ }^{8}$ and are used for the construction of a low-threshold laser in which the high field from the laser surrounds a void for chemical sensing or strong coupling to atomic light sources.

Our laser design uses the simplest triangular lattice single-defect cavity containing a fractional edge dislocation. The cavity consists of a defect hole that is smaller than surrounding holes which define the photonic crystal mirror. The row that contains the defect hole is elongated by moving the two photonic crystal half planes a fraction of a lattice constant apart in the $\Gamma X$ direction, introducing a dislocation with width $p$ (Fig. 1). We have shown earlier ${ }^{8}$ that by tuning this $p$ parameter, $Q$ factors of single-defect cavities are significantly improved, and can reach values of over 10000 when $p / a=10 \%$ ( $a$ is the lattice constant). These high- $Q$ values are obtained while maintaining a very small mode volume of $V_{\text {mode }} \approx 0.1(\lambda / 2) .{ }^{3}$ The cavity used in our laser was originally designed for cavity quantum electrodynamic experiments and nanospectroscopy. Light sources or absorbing molecules can be placed into the small hole within the center of the $2 \mathrm{D}$ photonic crystal cavity, where the optical field intensity is the strongest. On the other hand, it is clear that the presence of a hole at the point of maximum field intensity is not desirable in low-threshold laser designs, since the overlap with the gain region (e.g., quantum wells) is decreased. Therefore, we

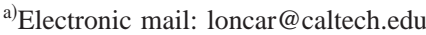

expect even better cavity designs to yield further improvements over the performance of the lasers described here.

Our structures are fabricated in InGaAsP quantum well material. Metalorganic chemical vapor deposition was used to grow the active laser structure on an InP substrate. Optical gain is provided by four $9 \mathrm{~nm}$ thick, compressively strained, quantum wells with an electronic band gap at $E_{g}$ $=1.55 \mu \mathrm{m}$, separated by $20 \mathrm{~nm}$ thick InGaAsP barriers $\left(E_{g}\right.$ $=1.22 \mu \mathrm{m})$. This active material is placed in the center of a $330 \mathrm{~nm}$ thick InGaAsP slab $\left(E_{g}=1.22 \mu \mathrm{m}\right)$, with a $1 \mu \mathrm{m}$ thick sacrificial InP layer underneath the slab. An InGaAs etch stop is introduced between the sacrificial layer and the InP substrate. The active quaternary material is designed to operate at $\lambda=1.55 \mu \mathrm{m}$, and because of the compressive strain, a coupling is strongest to the transverse electric (TE) polarized modes of the slab. This is desirable since in a triangular lattice photonic crystal, the band gap exists for TElike polarized light. An etch mask consists of $40 \mathrm{~nm} \mathrm{Au}$ evaporated on top of $100 \mathrm{~nm} \mathrm{SiON}$, deposited using plasmaenhanced chemical vapor deposition. The fabrication process starts with the deposition of $150 \mathrm{~nm}$ of poly(methylmethacrylate) (PMMA) electron beam resist, followed by electron-beam lithography to define structures in PMMA. We use $\mathrm{Ar}^{+}$ion milling to transfer the mask pattern through the $\mathrm{Au}$ metal mask, and follow this step by a $\mathrm{C}_{2} \mathrm{~F}_{6}$ reactive ion etching (RIE) to transfer the mask from the $\mathrm{Au}$ into the SiON. Cl-based inductively coupled plasma RIE is finally used to transfer the pattern from the SiON mask layer into

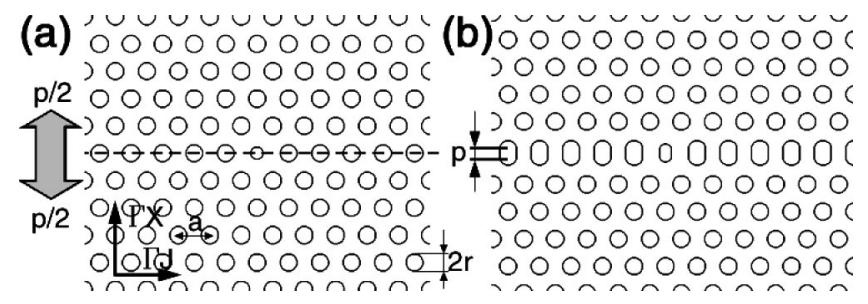

FIG. 1. (a) Conventional single defect cavity $(p=0)$. When structure is "cut" along the dashed line, and two PPC half planes are dislocated along $\Gamma X$ direction by $p / 2$ (b) high- $Q$ cavity can be formed $(p=0.25 \cdot a)$. 


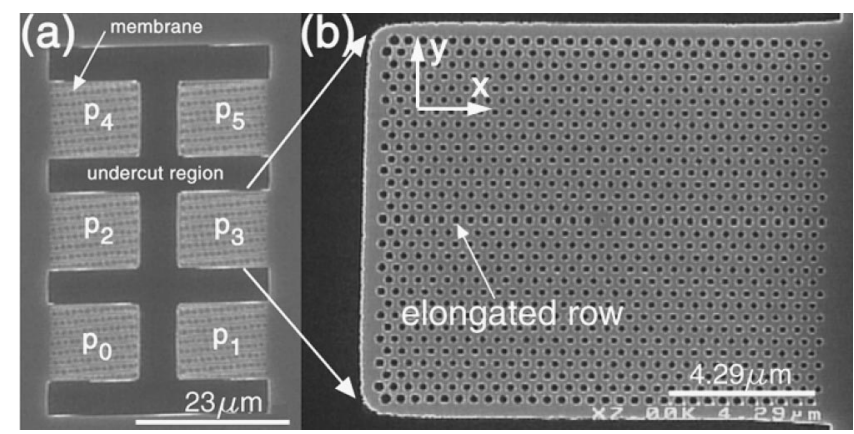

FIG. 2. (a) Six different cavities with elongation parameters $p_{0}=0, p_{1}$ $=0.05 \cdot a, \quad p_{2}=0.1 \cdot a, \quad p_{3}=0.15 \cdot a, \quad p_{4}=0.2 \cdot a$, and $p_{5}=0.25 \cdot a$. (b) Blowup of $p_{3}$ cavity.

the InGaAsP. Finally, the mask is removed in a hydrofluoric acid and the InGaAsP membrane is released from the substrate by wet etching in $4: 1 \mathrm{HCl}$ :water solution at $4{ }^{\circ} \mathrm{C}$. The final structure is a free standing membrane supported at one side (Fig. 2). Each pattern, shown in Fig. 2(a), consists of six different cavities that have received the same electron dose during the electron beam lithography step and, therefore, should have similar hole size $(r)$ and lattice constant $(a)$. The only difference between cavities within a pattern is the value of the elongation parameter $p$ that assumed values in the range $p / a \in(0,0.25)$. The periodicity of the structure characterized here was $a=435 \mathrm{~nm}$ and a hole radius $r$ $=138 \mathrm{~nm}$. This combination of geometries leads to a relative thickness of $d / a=0.759$ and relative hole size of $r / a$ $=0.317$. Using three-dimensional finite difference time domain code (3D FDTD), we have found that in this case, the photonic band gap is located in the range $a / \lambda \in(0.253$, $0.345)$.

The structures were optically pumped using 10-30 ns long pulses (periodicity $1 \mu \mathrm{s}$ ) from a semiconductor laser diode $\left(\lambda_{\text {pump }}=830 \mathrm{~nm}\right)$. The pump beam was focused through a $100 \times$ objective lens onto the sample surface to obtain a spot size of about $3 \mu \mathrm{m}$. The emission from the cavities is collected through the same lens, and the spectrum of the emitted light signal is detected with an optical spectrum analyzer. An additional flip-up mirror is used to obtain the optical images from the excitation pump spot and the cavity modes.

As the first step, we have measured the emission from the unprocessed InGaAsP material. We have found that emission exists between 1300 and $1650 \mathrm{~nm}$, with a maximum at around $1550 \mathrm{~nm}$. This wavelength range corresponds to normalized frequencies of $a / \lambda \in(0.264,0.335)$, which is within the band gap of the bulk photonic crystal mirrors surrounding the cavity. Next, we tested all six cavities (Fig. 2) in order to measure their resonant modes. We have found two prominent resonant peaks in the emission range of our InGaAsP material, and observed that these two modes are linearly polarized, but have orthogonal polarization (Fig. 3). This is in excellent agreement with our 3D FDTD analysis that shows that two orthogonally polarized modes (LQ polarized along the $y$ axis and HQ along the $x$ axis) exist in this wavelength range (Fig. 3). We have also found that the position of these resonances depends strongly on the value of the elongation parameter $p$ (Fig. 4), as predicted in our earlier publication. ${ }^{8,9}$ Moreover, theory predicts that the mode at Downloaded 18 Dec 2005 to 131.215.240.9. Redistribution subject
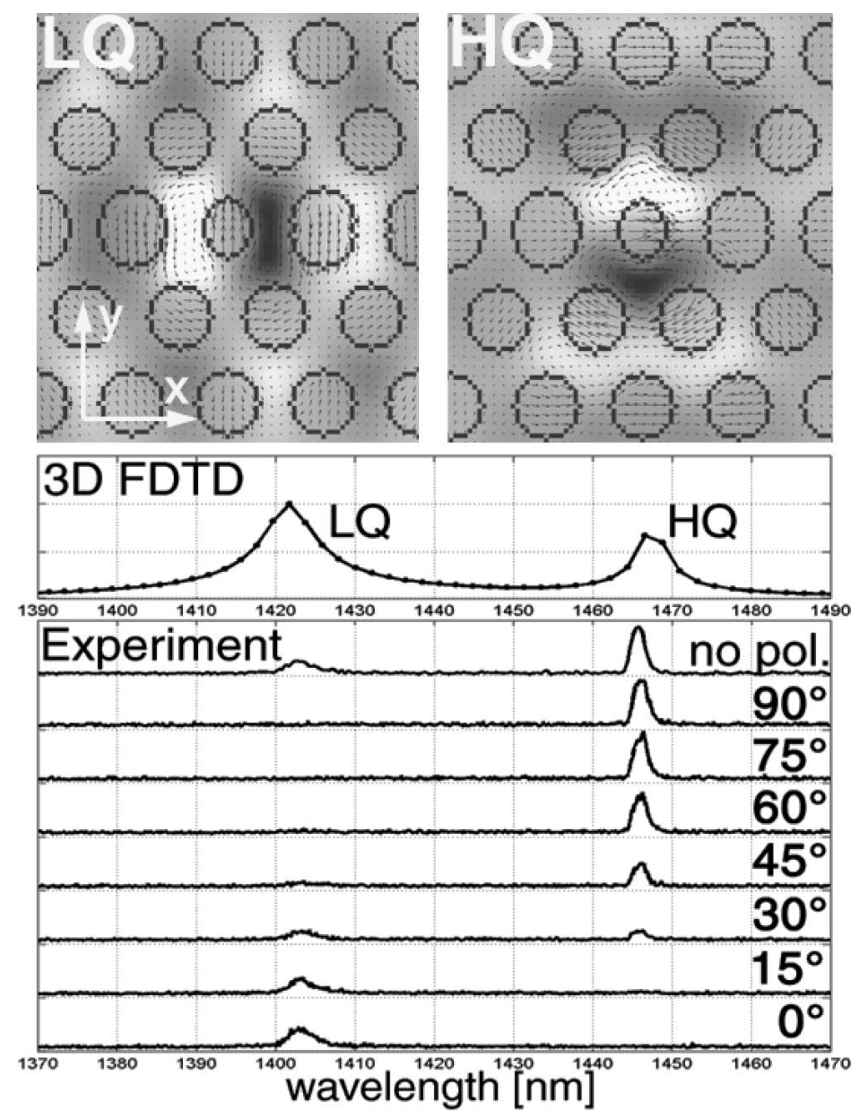

FIG. 3. Polarization dependance of the two modes detected in the $p_{3}$ cavity, obtained using polarizer positioned at different angles with respect to $y$-axis direction $\left(0^{\circ}\right.$ - polarization along $y$ axis $)$. Figure also shows mode profiles ( $B_{z}$ component) and polarization ( $\mathbf{E}$ field) of the LQ and HQ modes, the result of 3D FDTD analysis.

longer wavelengths (HQ) should have much higher $Q$ values than the one at shorter wavelengths (LQ). This was confirmed in our experimental measurements, and $Q$ values of approximately 2000 were found in the case of HQ modes ( $p_{5}$ cavity), while $Q$ s of only several hundreds were measured in the case of LQ modes.

Next, we tested our devices for lasing, by using pump pulses with a microsecond periodicity. Poor thermal heat sinking was expected for our membrane resonators, since the free standing membranes are attached to the substrate at only one side. Thus, we have pumped the structures with limited duty cycles ( $\mathrm{DC} \leqslant 3 \%$ ). Lasing was observed in our cavities for several $p_{4}$ and $p_{5}$ structures, with different $r / a$ parameters and defect hole sizes. In some cases, lasing could also be observed in $p_{3}$ cavities. In Fig. 5, we show the depen-

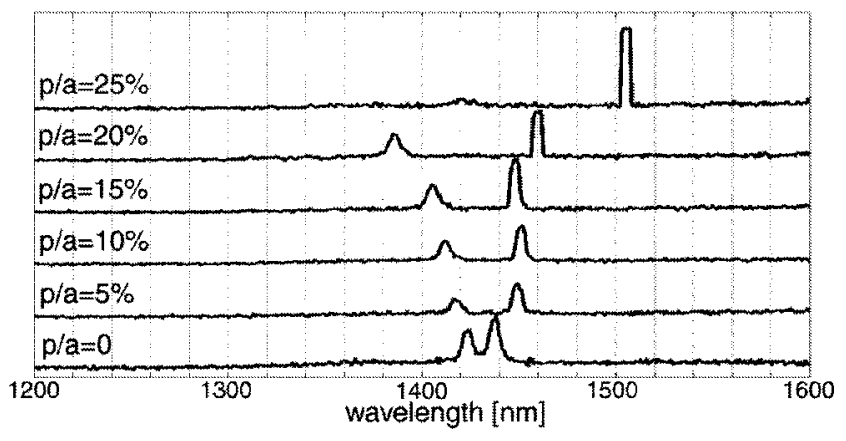

FIG. 4. Position of resonant modes detected in cavities $p_{0}-p_{5}$. Ficense or copyright, see http://apl.aip.org/apl/copyright.jsp
licens $p_{0}-p_{5}$. 


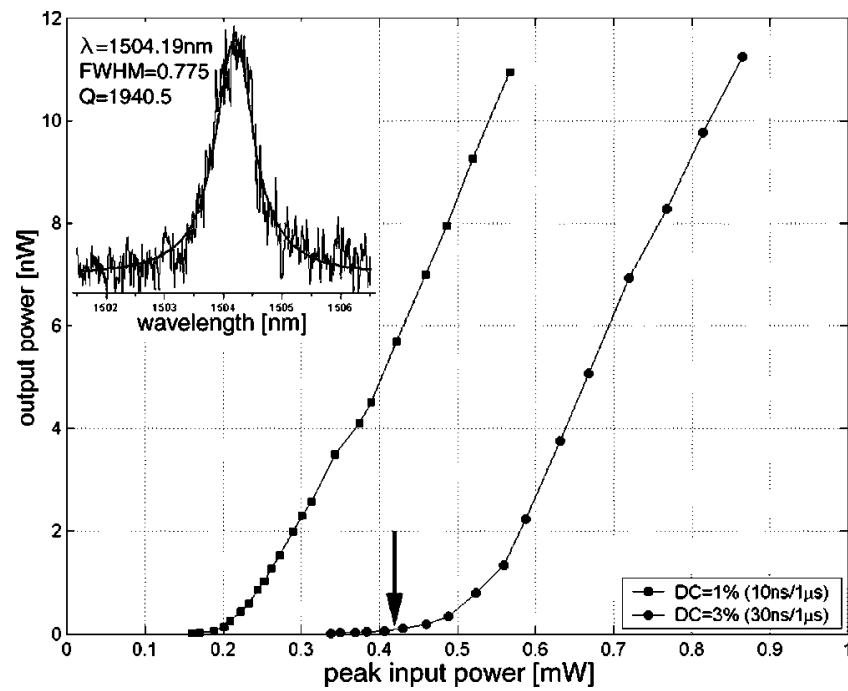

FIG. 5. $L-L$ curve for $p_{5}$ cavity for two different DCs. The pulse periodicity was $1 \mu \mathrm{s}$ in both cases. Spectrum taken below threshold (arrow) is shown in the inset.

dence of the output power on the input power ( $L-L$ curve) for one of the lasing photonic crystal nanocavities. Threshold powers as low as $P_{\mathrm{th}}=214 \mu \mathrm{W}$ were observed when this laser was pumped with approximately $1 \%$ duty cycle. ${ }^{11}$ To the best of our knowledge, this is the smallest threshold power observed in quantum well-based photonic crystal lasers. The quality factor in the case of the $p_{5}$ structure was estimated from below threshold luminescence measurements to be around $Q \approx 2000$ (Fig. 5), and this is in good agreement with theoretical predictions. Unfortunately, we were not able to obtain reliable estimates for $Q$-factor values in the case of $p_{2}$ and $p_{3}$ cavities due to the weak emission from these devices below threshold. However, according to theoretical predictions, we expect these cavities to have $Q$ factors as high as 10000 . Yoshie et al. ${ }^{10}$ have analyzed very similar devices in quantum dot material and have obtained $Q$ factors of about 2800 .

In Fig. 6, we show the $L-L$ curve for a laser structure with an elongation $p_{4}$. In addition to different elongation, the holes are smaller in this structure $(r / a=0.292)$ than in

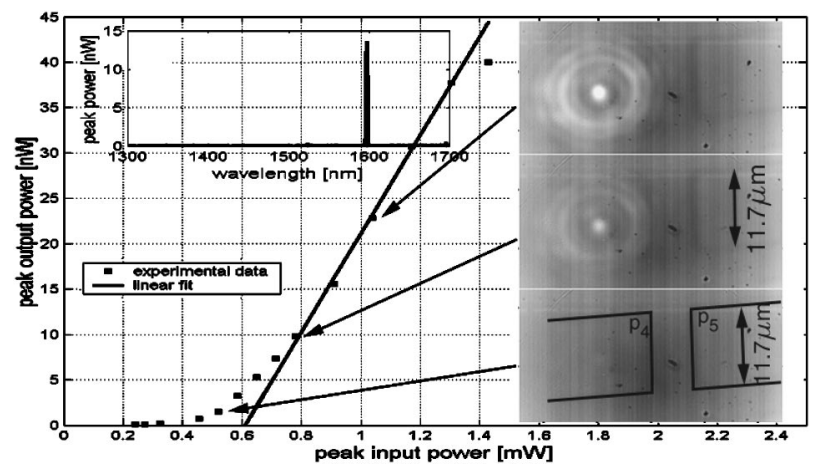

FIG. 6. $L-L$ curve for $p_{4}$ cavity $(r / a=0.292)$. Lasing action occurs at $\lambda$ $=1598 \mathrm{~nm}$. Insets show spectrum above threshold, and mode profiles of the lasing mode for several pump levels. The boundaries of the structure can also be seen. The mode is very well localized to the center of the cavity. the lasers described herein. This cavity was again found to support two modes, and the one at longer wavelength also has the higher- $Q$ factor which lases. A photoluminescence spectrum taken above threshold, as well as the mode profiles taken at different pumping levels, are shown in the inset of Fig. 6. It can be seen that the lasing mode is very well localized in the center of this cavity, and has a spot size on the order of $3.9 \mu \mathrm{m}^{2}$. This is an indication of a small mode volume of this laser. When the pump beam is only slightly moved from the center of the cavity (less than $1 \mu \mathrm{m}$ ), the strong light intensity shown in Fig. 6 disappears. This microluminescence pumping result is another confirmation that we indeed observe lasing from a well localized, small mode volume, high- $Q$ resonant mode.

In conclusion, we have observed room-temperature lasing from high- $Q$ cavities based on fractional edge dislocations in triangular lattice PPCs. Lasing is observed from the high- $Q$ dipole mode of this nanocavity. In spite of the unusual design of our structures, which have a hole etched through the position of maximum field intensity and therefore reduced overlap with gain material, we observe lowthreshold powers in our devices. We have attributed this to the small mode volume and the high- $Q$ factors inherent to our device design. Polarization and lithographic tuning properties of high- and low- $Q$ modes are in an excellent agreement with theoretical FDTD predictions. The mode profile taken by our IR camera shows that the lasing resonance is well localized to the center of our cavity. Based on these experimental results, we conclude that the observed lasing corresponds to the high- $Q$ mode of our fractional edge dislocation cavity.

The authors would like to thank William Green from Caltech for many valuable discussions. This work was supported by the NSF under Grants Nos. ECS-9912039 and DMR-0103134, and the AFOSR Contract No. F49620-01-10497. Two of the authors (P.G. and Y.Q.) would like to acknowledge the partial support from the Cross Enterprise Technology Development Program at the Jet Propulsion Laboratory (under a contract with the National Aeronautics and Space Administration).

\footnotetext{
${ }^{1}$ E. Yablonovitch, Phys. Rev. Lett. 58, 2059 (1987).

${ }^{2}$ T. F. Krauss, R. M. De La Rue, and S. Brand, Nature (London) 383, 699 (1996).

${ }^{3}$ O. J. Painter, A. Husain, A. Scherer, J. D. O'Brien, I. Kim, and P. D. Dapkus, J. Lightwave Technol. 17, 2082 (1999).

${ }^{4}$ H. G. Park, J. K. Hwang, J. Huh, H. Y. Ryu, and Y. H. Lee, Appl. Phys. Lett. 79, 3032 (2001).

${ }^{5}$ P. T. Lee, J. R. Cao, S. J. Choi, Z. J. Wei, J. D. O'Brien, and P. D. Dapkus, IEEE Photonics Technol. Lett. 14, 435 (2002).

${ }^{6}$ H. Y. Ryu, S. H. Kim, H. G. Park, J. K. Hwang, and Y. H. Lee, Appl. Phys. Lett. 80, 3883 (2002).

${ }^{7}$ C. Monat, C. Seassal, X. Letartre, P. Viktorovitch, P. Regreny, M. Gendry, P. Rojo-Romeo, G. Hollinger, E. Jalaguier, S. Pocas, and B. Aspar, Electron. Lett. 37, 764 (2001).

${ }^{8}$ J. Vučković, M. Lončar, H. Mabuchi, and A. Scherer, Phys. Rev. E 65, 016608 (2001).

${ }^{9}$ J. Vučković, M. Lončar, H. Mabuchi, and A. Scherer, IEEE J. Quantum Electron. 38, 850 (2002).

${ }^{10}$ T. Yoshie, J. Vučković, A. Scherer, H. Chen, and D. Deppe, Appl. Phys. Lett. 79, 4289 (2001).

${ }^{11}$ Recently, we have measured threshold powers as low as $110 \mu \mathrm{W}$ in simi-
} lar $p_{5}$ structures. 